\title{
Acupuncture and Related Therapies for Obesity: A Network Meta-Analysis
}

\author{
Yanji Zhang $\left(\mathbb{D},{ }^{1}\right.$ Jia Li $\mathbb{D},{ }^{1,2,3}$ Guoyan Mo, ${ }^{4}$ Jing Liu, ${ }^{1}$ \\ Huisheng Yang, ${ }^{5}$ Xianglin Chen $\mathbb{D},{ }^{1}$ Hui Liu $\mathbb{D},{ }^{1}$ Teng Cai $\mathbb{D},{ }^{1}$ Xian Zhang $\mathbb{D},{ }^{1}$ \\ Xiangmin Tian $\mathbb{D}^{1},{ }^{1}$ Zhongyu Zhou $\mathbb{D}^{2,3}$ and Wei Huang $\mathbb{D}^{2,3}$ \\ ${ }^{1}$ College of Acupuncture and Orthopedics, Hubei University of \\ Chinese Medicine/Hubei Provincial Collaborative Innovation Center of Preventive Treatment by \\ Acupuncture and Moxibustion, Wuhan, China \\ ${ }^{2}$ Department of Acupuncture, Hubei Provincial Hospital of Traditional Chinese Medicine, Wuhan, China \\ ${ }^{3}$ Hubei Province Academy of Traditional Chinese Medicine, Wuhan, China \\ ${ }^{4}$ China Key Laboratory of TCM Resource and Prescription, Hubei University of Chinese Medicine, \\ Ministry of Education, Wuhan, China \\ ${ }^{5}$ Institute of Acupuncture and Moxibustion of China Academy of Chinese Medical Sciences, Beijing, China
}

Correspondence should be addressed to Zhongyu Zhou; 2209447940@qq.com and Wei Huang; huangwei@hbhtcm.com

Received 21 August 2018; Accepted 9 September 2018; Published 30 September 2018

Academic Editor: Mariangela Rondanelli

Copyright (C) 2018 Yanji Zhang et al. This is an open access article distributed under the Creative Commons Attribution License, which permits unrestricted use, distribution, and reproduction in any medium, provided the original work is properly cited.

\begin{abstract}
Obesity is a worldwide public health problem. Currently, increasing evidence suggests acupuncture and related therapies are effective for obesity. This network meta-analysis (NMA) was performed to compare the effectiveness of different acupuncture and related therapies. We searched potential randomized controlled trials (RCTs) in three international databases. Thirty-four trials involving 2283 participants were included. Pairwise meta-analysis showed that acupuncture and related therapies were superior to lifestyle modification and placebo in reducing weight and body mass index (BMI). Based on decreases in body weight, results from NMA showed that acupoint catgut embedding (standard mean difference [SMD]: 1.26; 95\% credible interval [95\% CI], 0.46-2.06), acupuncture (SMD: 2.72; 95\% CrI, 0.06-5.29), and combination of acupuncture and related theories (SMD: 3.65; 95\% CrI, 0.96-6.94) were more effective than placebo. Another NMA result indicated that acupoint catgut embedding (SMD: 0.63; 95\% CI, 0.25-1.11), acupuncture (SMD: 1.28; 95\% CrI, 0.43-2.06), combination of acupuncture and related therapies (SMD: 1.44; 95\% CrI, 0.64-2.38), and electroacupuncture (SMD: 0.60; 95\% CrI, 0.03-1.22) were superior to lifestyle modification in decreasing BMI. Combination of acupuncture and related therapies was ranked the optimal method for both reducing weight and BMI. Further studies will clarify which combination of acupuncture and related therapies is better.
\end{abstract}

\section{Introduction}

Obesity, a worldwide public health problem, is described as an adiposity-based chronic disease [1]. Currently, guidelines recommended using body mass index (BMI) to classify individuals as having obesity $\left(\mathrm{BMI} \geqslant 30 \mathrm{~kg} / \mathrm{m}^{2}\right)$ [2]. Based on the survey conducted previously, the standardized prevalence rates for obesity in adult were $34.9 \%$ in United States and $17.7 \%$ in China [3, 4]. Moreover, it is associated with other health concerns, such as insulin resistance, type 2 diabetes mellitus, cardiovascular disease, and cancer, which increased individuals and societies' medical burden [5].

Lifestyle modification, pharmacotherapy, and bariatric surgery are considered the mainstay of therapy for obesity [2]. Although diet and exercise play an essential role in the weight management, their precise mode of action remains controversial [6]. Five long-term medicines (naltrexonebupropion, phentermine-topiramate orlistat, lorcaserin, and liraglutide) have been approved by US Food and Drug Administration (FDA) for the treatment of obesity [7]. The 
latest research suggested that phentermine-topiramate was associated with the highest possibility of achieving at least 5\% weight loss [7]. However, little is known about the long-term safety profile of pharmacotherapy for weight loss. The effectiveness of bariatric procedures for treating obesity has been reported in several randomized controlled trials (RCTs) [810]. Nevertheless, the evidence on cardiovascular disease and mortality remains to be validated [11]. Therefore, it is necessary to explore other forms of alternative therapies which are both safe and effective in preventing gaining weight.

In reviewing the literature, it became evident that acupuncture and related therapies have been wildly used for obesity treatment. As mentioned in the meta-analysis, combination of acupuncture and lifestyle modification is more effective compared with lifestyle modification alone [12]. Results of Yeh's research suggested that ear acupoint stimulation had remarkable improvements in the anthropometric parameters of Body Weight (BW), BMI, and so on [13]. In addition, another systematic review performed in 2015 has also shown that clinical efficacy of acupoint catgut embedding therapy was better than that of the control group for simple obesity [14]. However, a major problem is that whether acupuncture or acupuncture-related therapies alone or combined therapy is more effective than lifestyle modification management remains disputable.

By using the technique of network meta-analysis (NMA), both direct and indirect randomized data can be analyzed, and recommended rankings of different treatments can be provided $[15,16]$. Therefore, we conducted this Bayesian network meta-analysis to analyse both direct and indirect comparisons of acupuncture and related methods for treating obesity. In this paper, changes in BW, BMI, and the rates of complications of included studies were analyzed.

\section{Methods}

Our research was conducted following the Preferred Reporting Items for Systematic Reviews and Meta-Analyses for Network Meta-Analysis (PRISMA-NMA) checklist [17] (see Appendix 1).

2.1. Data Sources and Search Strategy. Three electronic international databases (PubMed/Medline, Embase, and the Cochrane Library) were searched for potential RCTs (randomized controlled trials). We identified articles published from initiation to December 2017 with a limit to studies of RCT and without limitations on language or the form they are published in. The complete search strategies are shown in Appendix 2.

2.2. Study Selection. Two researchers (XC and HL) independently identified irrelevant research based on titles and abstracts. Additionally, full-text articles were scanned by these two researchers to identify eligible studies. All disagreements were resolved by consensus and adjudged by a third reviewer (TC) if necessary. In case of duplicate citations, the most updated studies were selected for data extraction.

2.3. Inclusion and Exclusion Criteria. The studies included in the NMA met the following criteria: (1) the study design must be a randomized controlled clinical trial (RCT); (2) patients diagnosed with simple obesity irrespective of ages and sex as study subjects; diagnostic criteria must be clear and inclusion and exclusion criteria were explicit; (3) at least one of the following efficacy outcomes or safety endpoints was included: BW, BMI, and adverse events; (4) participants in the experimental group have received acupuncture and related treatments (specifically, classical body acupuncture; electroacupuncture auricular acupoint stimulation; acupoint catgut embedding and warming acupuncture) alone or in combination; (5) English or Chinese language studies.

The following were excluded: (1) self-control and nonRCTs; (2) preclinical studies, systematic reviews, case reports, and meta-analyses; (3) reports without sufficient and clear original data; (4) participants having received other forms of acupuncture such as transcutaneous electrical nerve stimulation or laser acupuncture; (5) duplicate studies and studies reporting the same results.

2.4. Data Collection and Quality Assessment. According to a standard data collection sheet, two investigators (TC and $\mathrm{XZ}$ ) independently extracted the following data: (1) main characteristics of included randomized controlled trials (i.e., year of publication, type of intervention, patients characteristics, types of outcome, and reported adverse events); (2) details of acupuncture and related interventions (i.e., frequency and duration of acupuncture sessions, names of acupuncture points used, and retention time); (3) clinical outcome (i.e., summaries of mean, standard difference, and sample size between treatment groups). In some trials, the change between baseline and after treatment was failed to present. Using the methods recommended in the Cochrane Handbook for Systematic Reviews of Interventions (version 5.1) [18], the missing data was estimated using the following formula:

$$
\begin{aligned}
\bar{X}_{\text {change }} & =\bar{X}_{\text {post-treatment }}-\bar{X}_{\text {baseline }} \\
S D_{\text {change }} & =\sqrt{\left(S D_{\text {baseline }}\right)^{2}+\left(S D_{\text {post-treatment }}\right)^{2}-2 \times r \times S D_{\text {baseline }} \times S D_{\text {post-treatment }}}
\end{aligned}
$$

where $\mathrm{r}$ is a correlation coefficient with a value of 0.5 [19]. For each included RCT, two researches (XT and XC) independently assessed their risk of bias by the Cochrane Collaboration tool [20]. Bias risks of each study were assessed 
from six aspects: random sequence generation, allocation concealment, blinding of participants and investigators, blinding of outcome assessment, incomplete outcome data addressed, and selective outcome reporting, while ranked in high risk, low risk, and unclear risk.

2.5. Statistical Analysis. Firstly, standard pairwise meta-analysis was initially performed using the Review Manager (Version 5.3, Cochrane Collaboration, Oxford, UK). We calculated I-square $\left(\mathrm{I}^{2}\right)$ test to assess heterogeneity among RCTs [21]. To be specific, when there was $\mathrm{I}^{2}>50 \%$, they were analysed using a random effects model; otherwise, a fixed effect model was chosen. Subgroup analyses were conducted according to the type of acupuncture treatment and the treatment of control group. Mean difference (MD) with 95\% confidence intervals (CI) was used to analyze continuous data. We generated forest plots to illustrate the relative strength of curative effects.

Second, to indirectly compare the effectiveness among treatments of acupuncture and related therapies, we did a random effects model NMA within a Bayesian framework, by using WinBUGS (Version 1.4.3, MRC Biostatistics Unit, Cambridge, UK) $[22,23]$. Models were computed with Markov chain Monte Carlo (MCMC) simulation methods, using four chains with overdispersed initial values. We utilized the Markov chains for 50,000 simultaneous iterations after the first 20000 iterations were discarded because they may have an influence on the arbitrary value. In this process, the convergence of the model was assessed by the Brooks-GelmanRubin (BGR) method; a value of potential scale reduction factor (PSRF) close to 1 indicated the better convergence [24]. The continuous outcome was measured by a standard mean difference (SMD) with a 95\% credible intervals (CrI) for indirect comparisons.

Finally, plot of surface under the cumulative ranking curve (SUCRA) was generated using the STATA software (Version 13.0; Stata Corporation, College Station, Texas, USA), which indicated the probability of each intervention of being ranked best [25]. In our study, higher SUCRA scores mean the higher rank of the treatment [15]. A $\mathrm{Z}$ value and its corresponding $\mathrm{p}$-value were calculated, and an $\mathrm{R}$ value less than 0.05 indicated a statistically significant difference.

\section{Results}

3.1. Study Search. We performed this research on Dec 262017. As shown in Figure 1, a total of 1050 records were initially identified from the databases. 675 studies left after duplicates were removed. 577 records were excluded after carefully scanning titles and abstracts. Finally, 34 trials with 2283 participants were included in our NMA [26-59], covering 8 groups, manual acupuncture; electroacupuncture; auricular acupoint stimulation; acupoint catgut embedding; pharmacotherapy; warming acupuncture; lifestyle modification; placebo.

3.2. Study Description. Main characteristics of included RCTs were shown in Table 1. The participants were from Australia [28], the United States [26], Turkey [46], Korea [51], Iran
[36], Egypt [48], and China. Age of participants ranged from 15 to 70 years, while the sample size of the studies ranged from 12 to 86. Among the included RCTs, there were one four-arm trials, 5 three-arm trials, and 28 two-arm trials. Fourteen studies compared acupuncture to placebo. Ten studies compared acupuncture to lifestyle intervention. Six studies compared combined therapies to acupuncture alone. Details about acupuncture points used, retention time, frequency, and duration of acupuncture sessions were shown in Table 2. In these research, 30 articles [26-30, 32, 34-38, 4159] reported the weight loss, while 25 articles reported the change in BMI. The details of mean, standard difference (SD), and sample size between different groups for eligible studies are summarized in Appendix 3. The Cochrane risk of bias assessment was presented in Table 3. Furthermore, the network plot of included comparisons was shown in Figure 2.

\subsection{Pairwise Meta-Analyses}

3.3.1. Body Weight. A direct pairwise meta-analysis showed that acupuncture and related therapies showed a greater BW reduction than lifestyle modification (MD: 1.66; 95\% Confidence interval, 0.63to2.70) and placebo (MD: 1.15; 95\% CI, 0.67to1.63). When compared to acupuncture, combination of acupuncture and related theories showed a marginally stronger effect in weight loss (MD: 1.56; 95\% CI, 0.07to3.05). There was no statistically significant difference between combination of acupuncture and related theories and pharmacotherapy in their effectiveness in BW (MD: 2.44; 95\% CI, -1.98to6.86). (Table 4)

BMI. As for the comparison in reducing BMI, acupuncture and related therapies were found to be marginally superior to lifestyle modification (MD: 1.17; 95\% CI, 0.09to2.26) and placebo (MD: 0.57; 95\% CI, 0.40to0.74). The remaining direct comparisons did not show significant differences (Table 4).

\subsection{Network Meta-Analysis}

3.4.1. Body Weight. The NMA showed that all treatments other than acupuncture combined lifestyle modification were more efficacious than lifestyle modification. Three treatments were significantly more effective than placebo. Specifically, acupoint catgut embedding (SMD: 1.26; 95\% credible interval, 0.46to2.06), acupuncture (SMD: 2.72; 95\% CrI, 0.06to5.29), and combination of acupuncture and related therapies (SMD: 3.65; 95\% CrI, 0.96to6.94). Furthermore, moxibustion with warming needle was associated with a significantly improvement than lifestyle modification (SMD: -5.24; 95\% CrI, -10.15to-0.55) (Table 5).

3.4.2. BMI. Four treatments showed superiority over placebo, including acupoint catgut embedding (SMD: 1.31; 95\% CrI, 0.36to2.06), acupuncture (SMD: 1.94; 95\% CrI, 0.83to3.00), combination of acupuncture and related theories (SMD: 3.65; 95\% CrI, 0.96to6.94), and electroacupuncture (SMD: $1.28 ; 95 \% \mathrm{CrI}, 0.43$ to2.11). Four treatments were significantly more effective than lifestyle modification, including acupoint catgut embedding (SMD: 0.63; 95\% CI, 

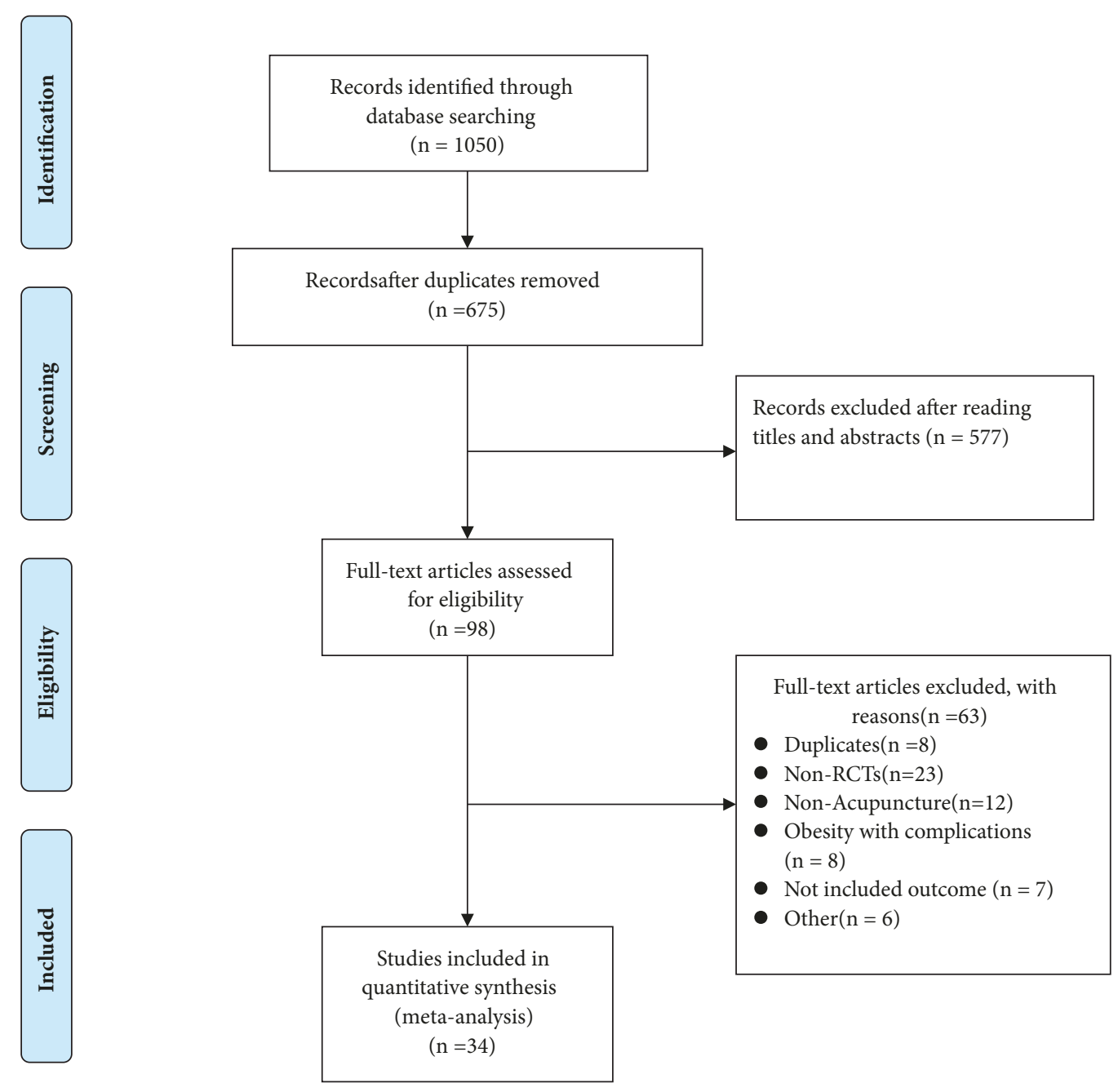

FIGURE 1: PRISMA flow chart.

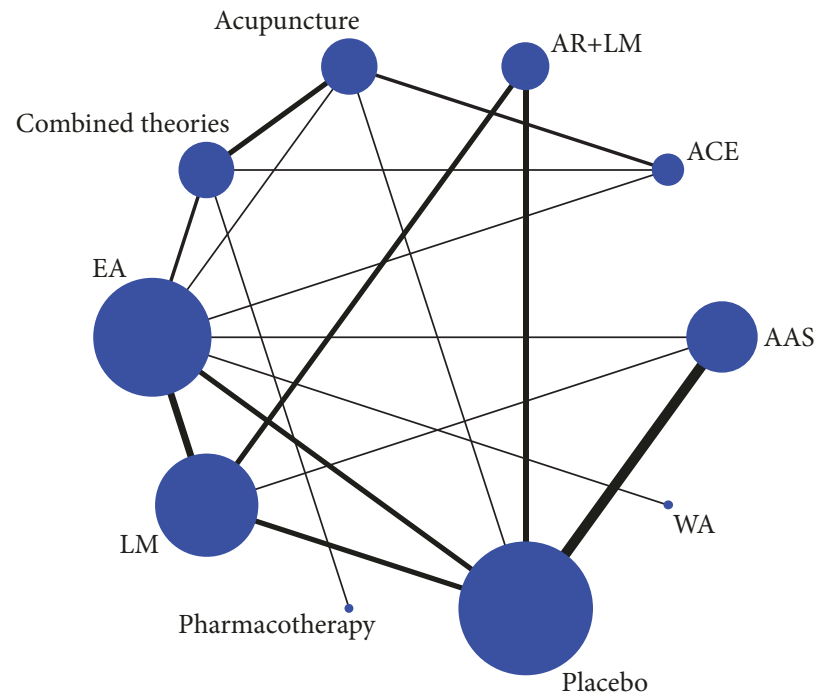

Body weight

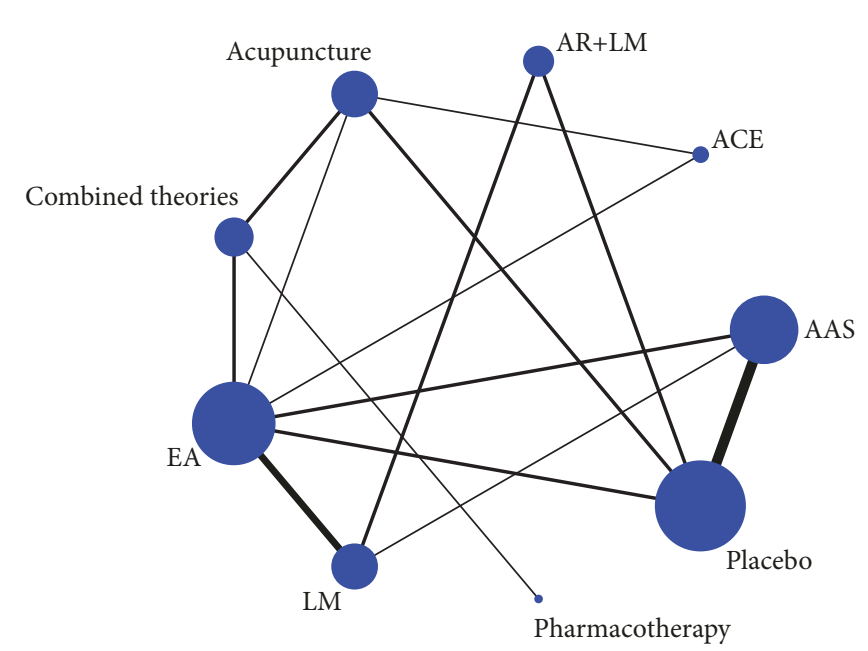

BMI

FIGURE 2: Network plot. BMI: body mass index; LM: lifestyle modification; AAS: auricular acupoint stimulation; EA: electroacupuncture; ACE: acupoint catgut embedding; WA: warming acupuncture; AR: acupuncture and related therapies; combined therapies: combination of acupuncture and related therapies. 


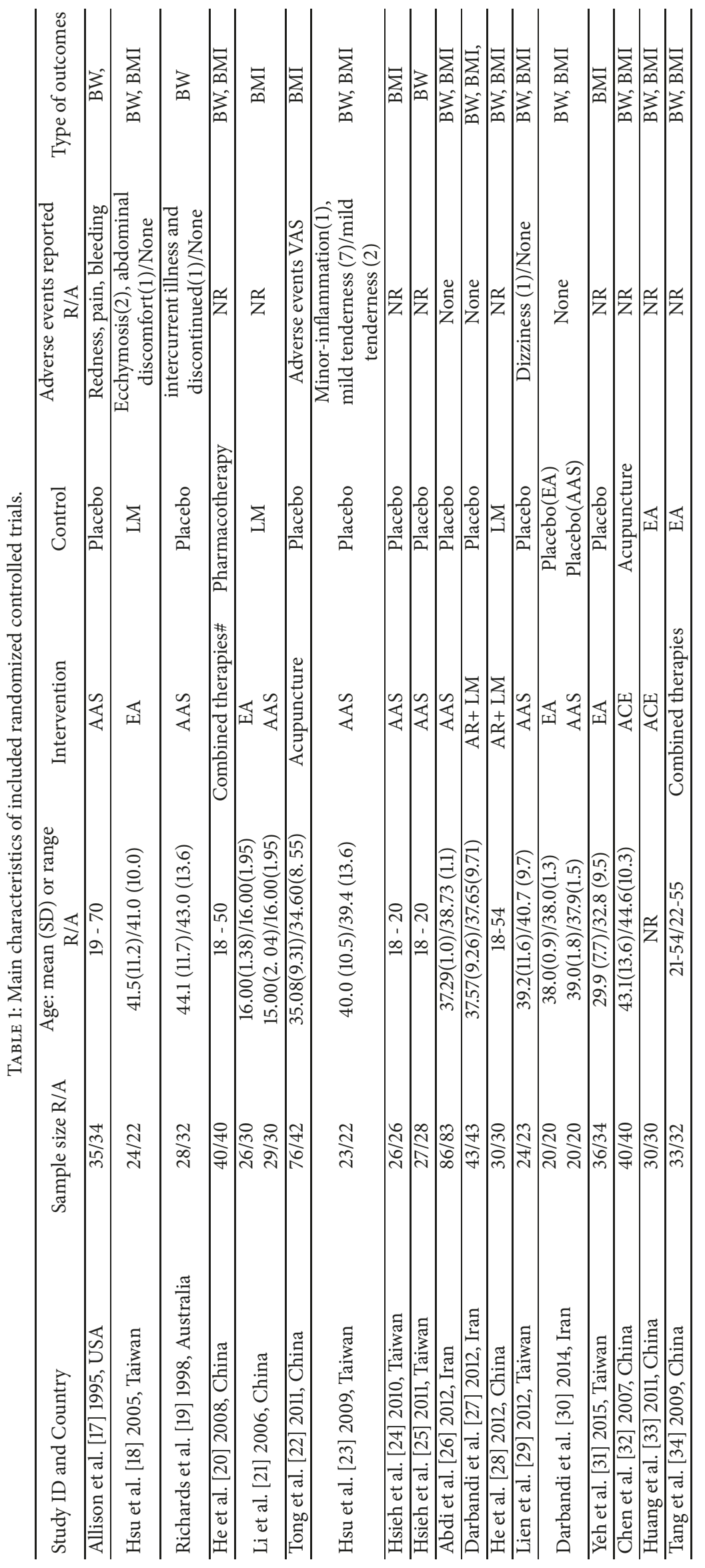




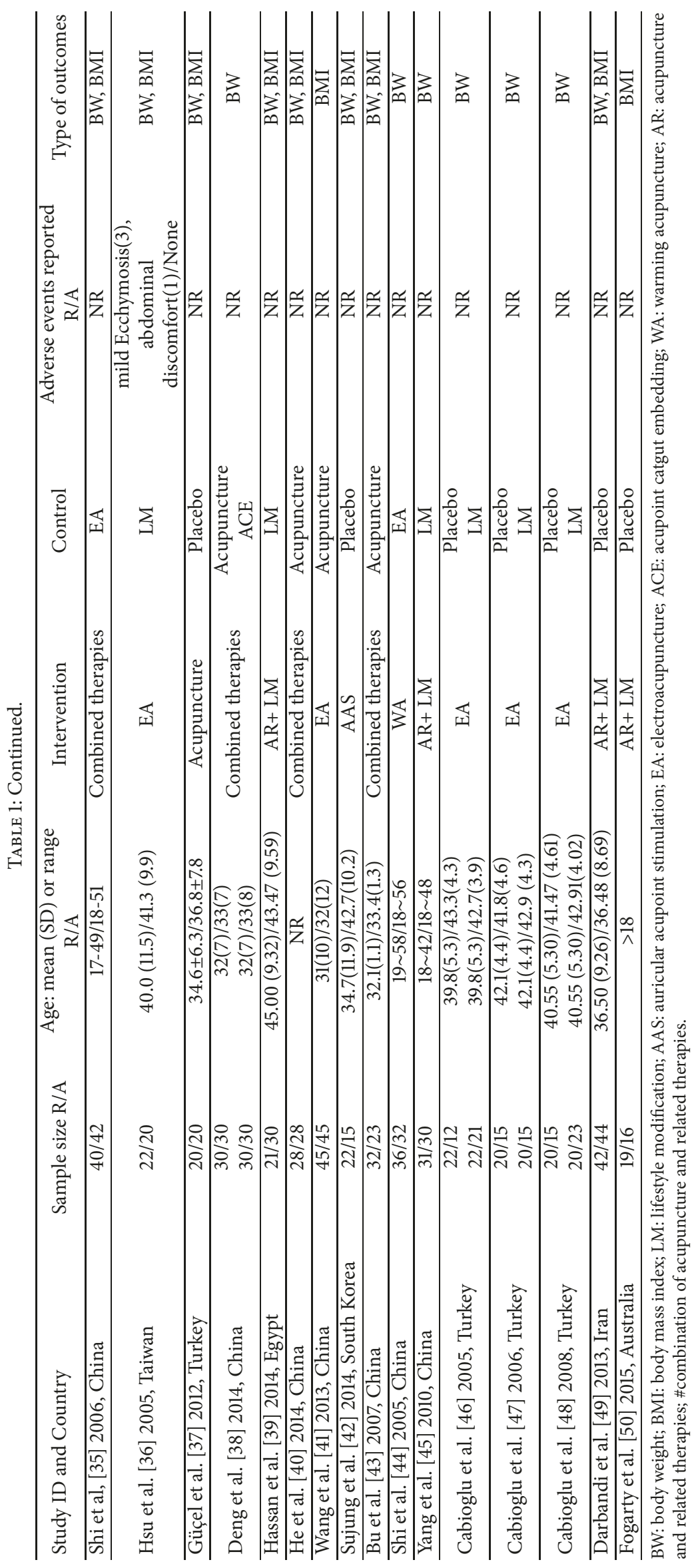




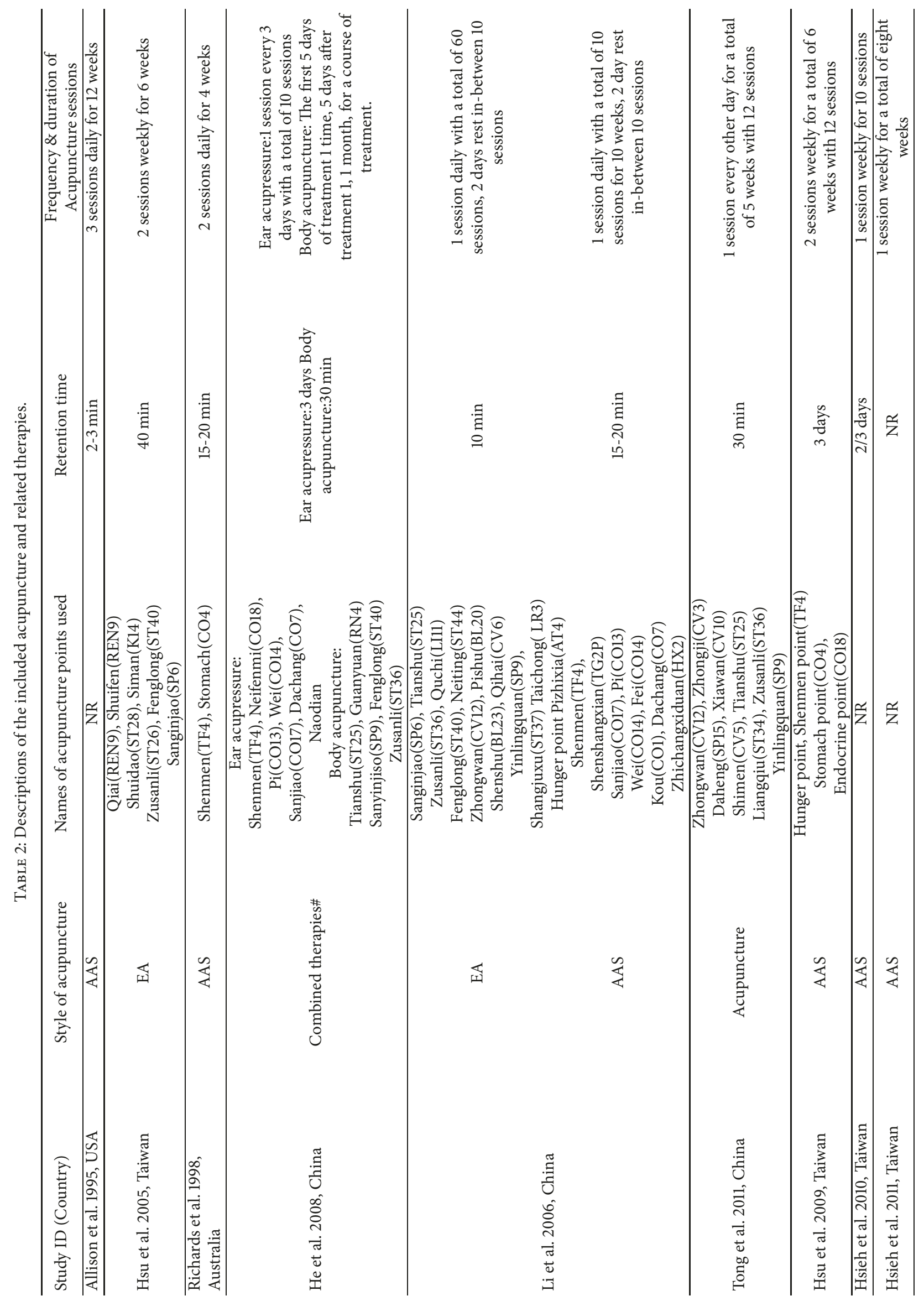




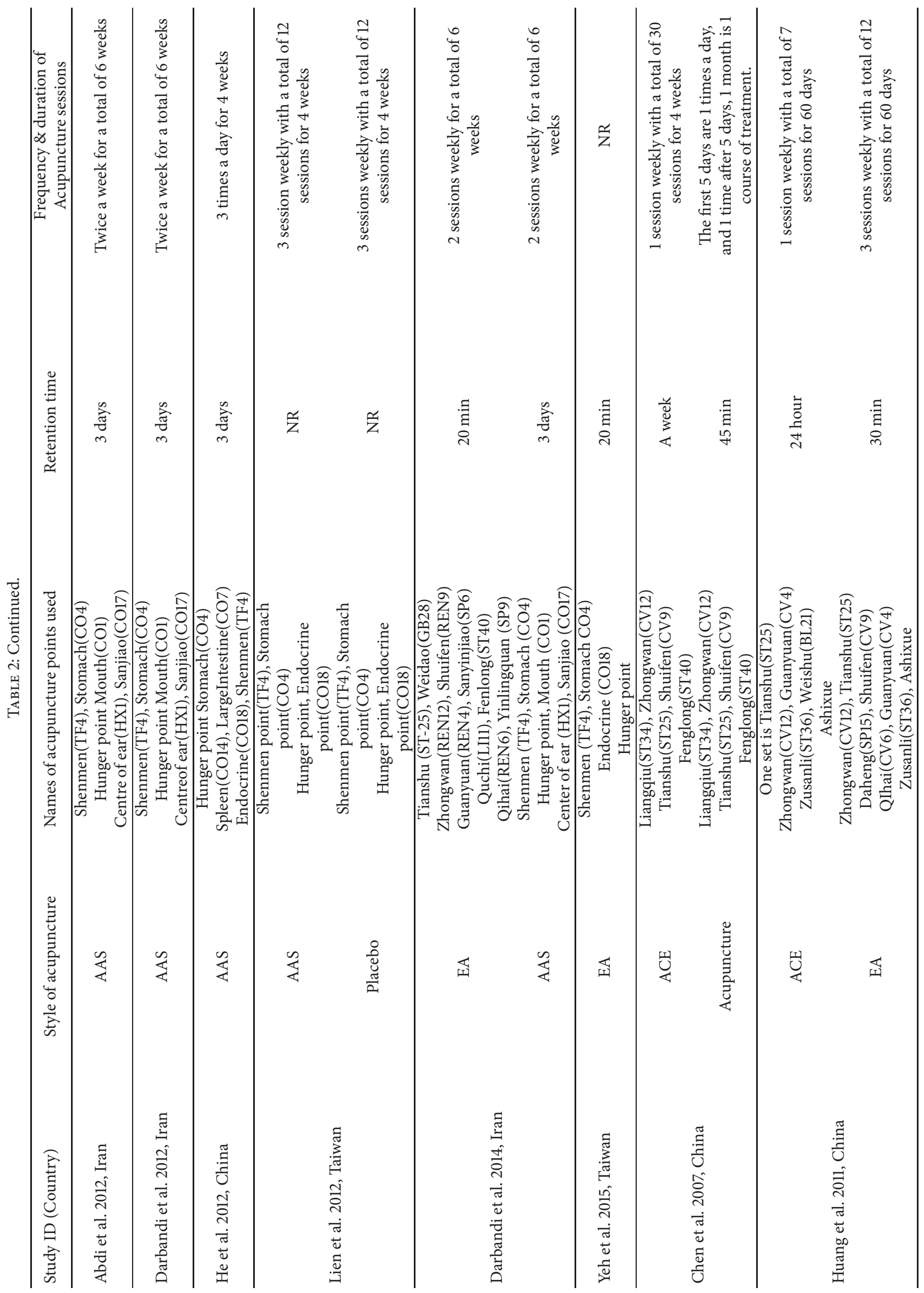




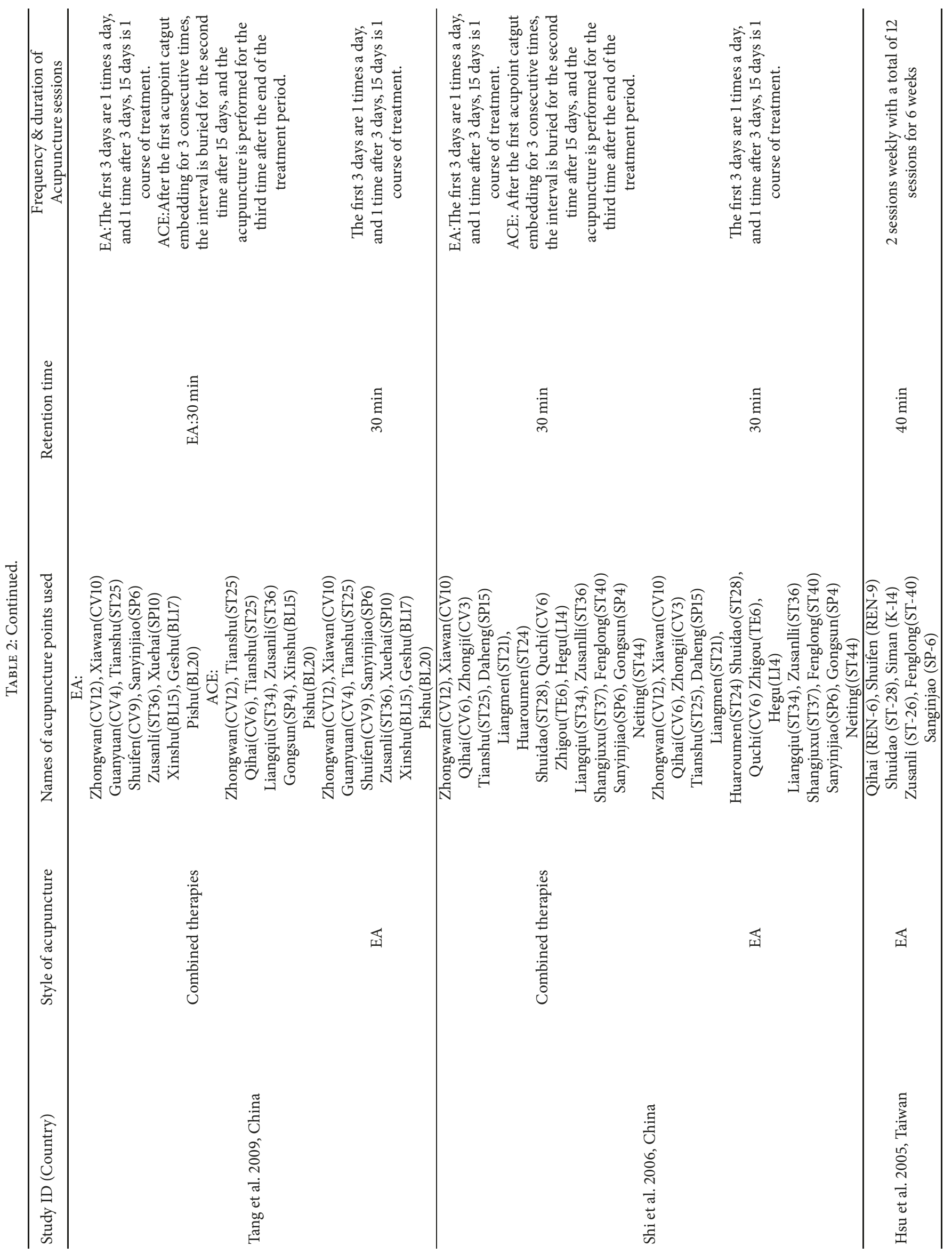




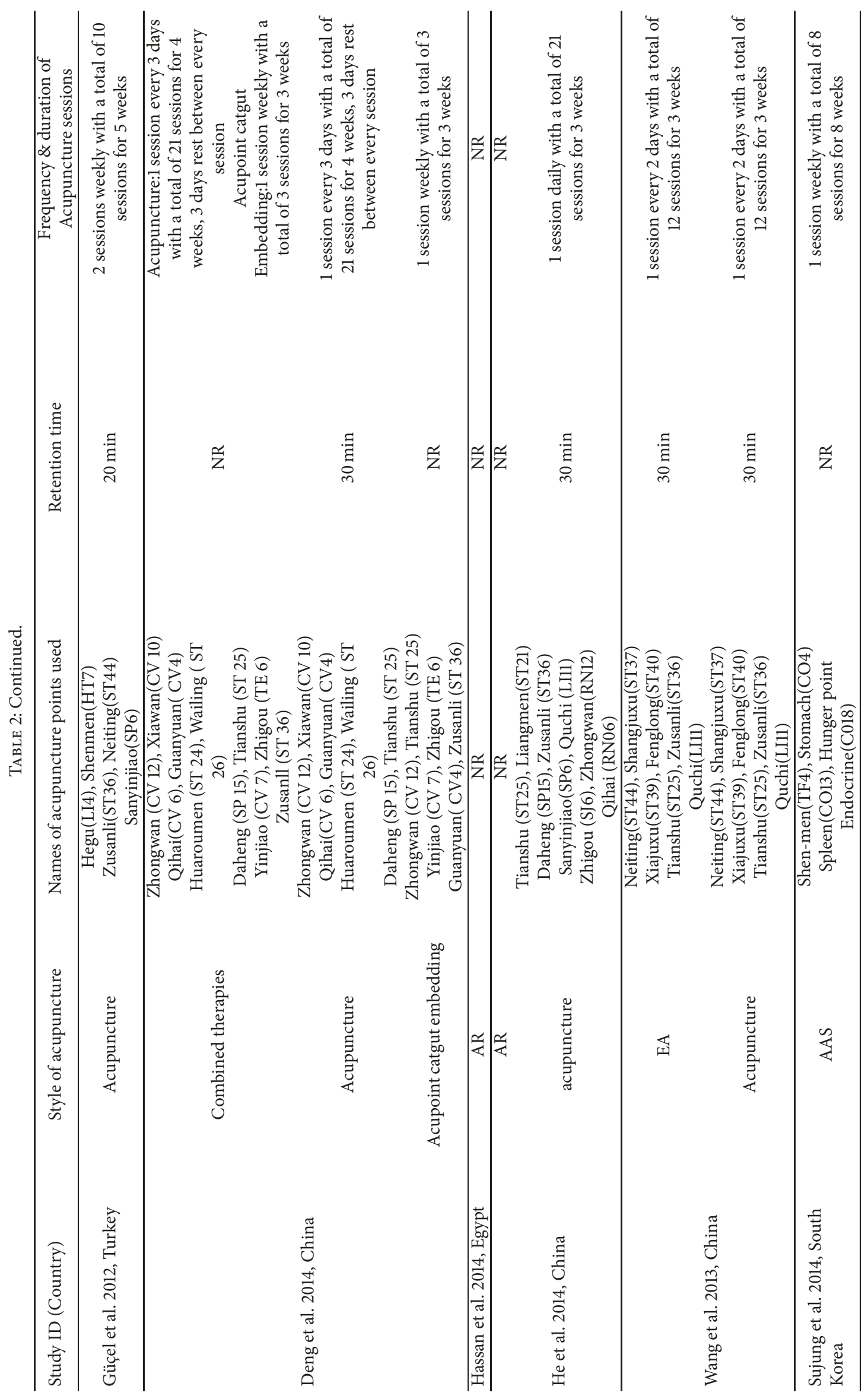




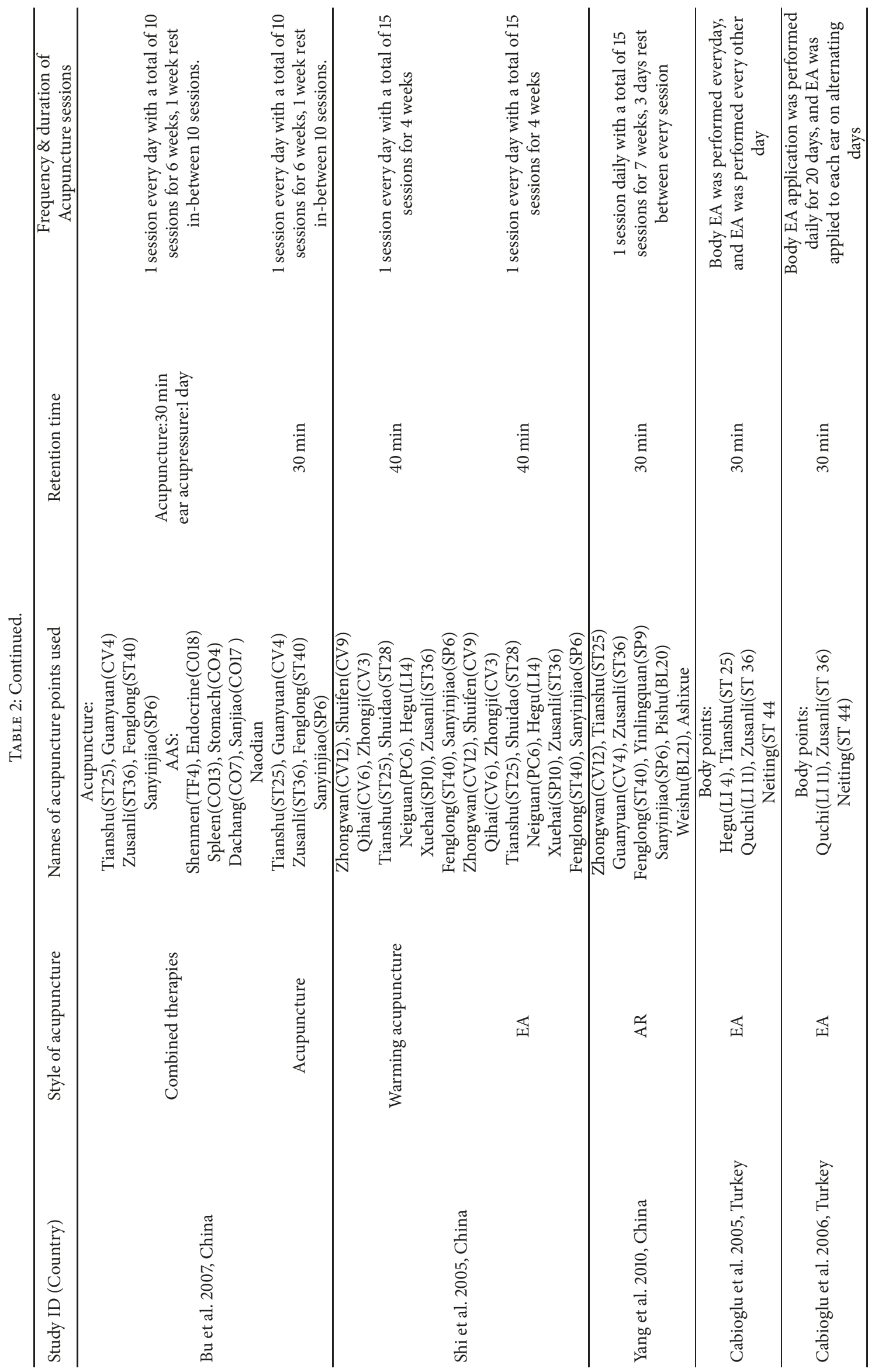




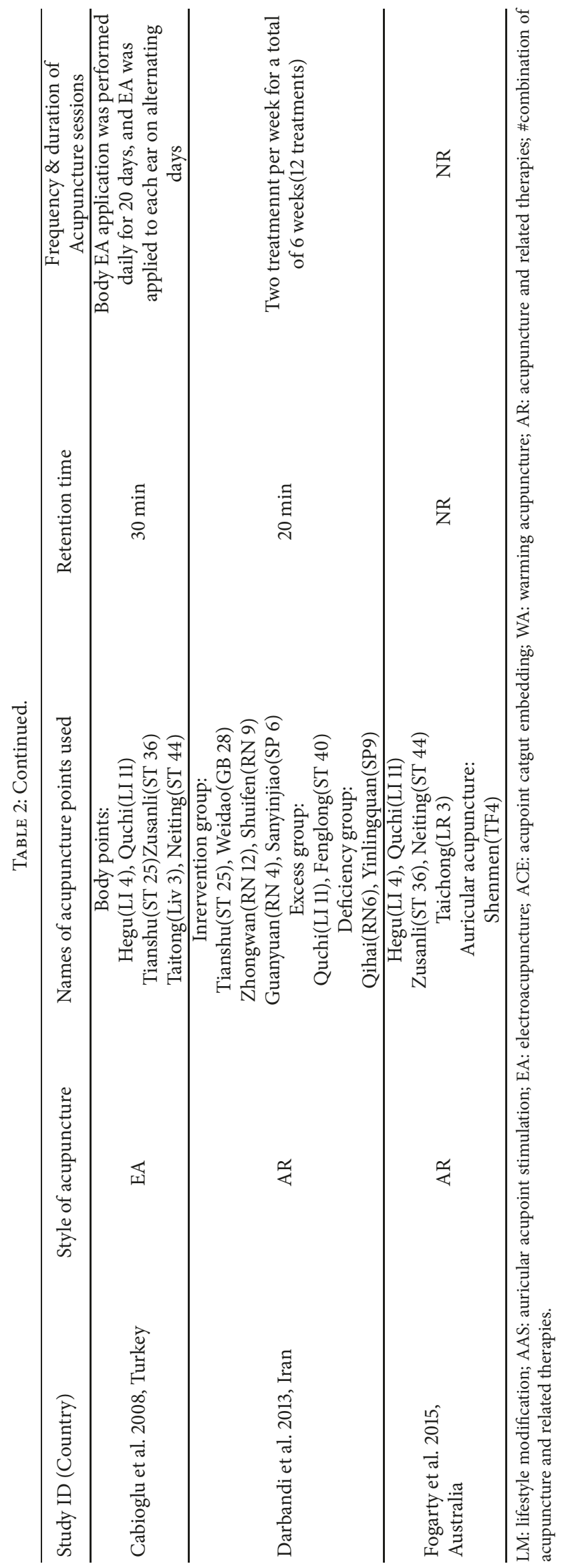




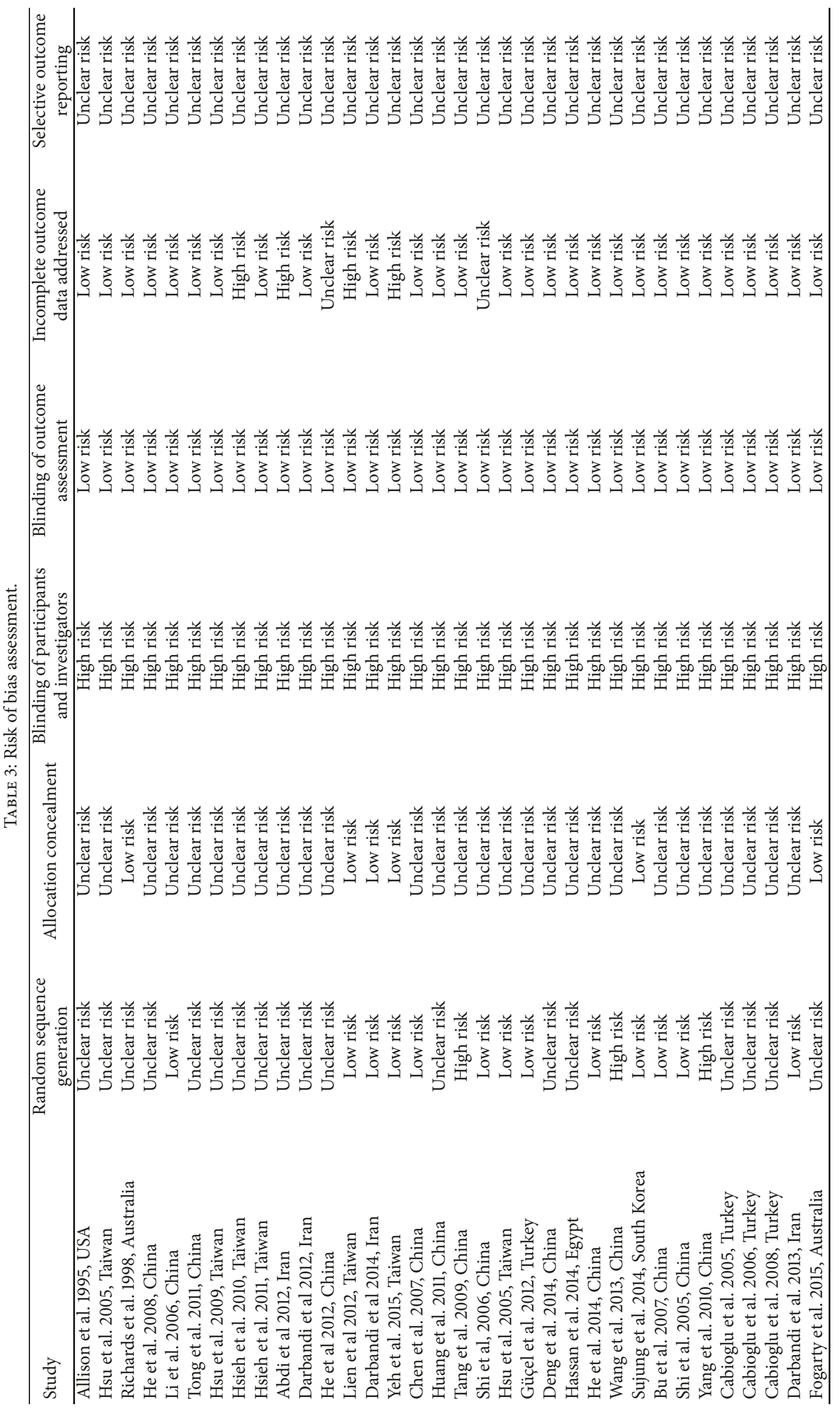


TABle 4: Pairwise meta-analyses.

\begin{tabular}{|c|c|c|c|c|c|}
\hline \multirow{2}{*}{ Comparison } & \multirow{2}{*}{ Pairwise OR (95\% CI) } & \multirow{2}{*}{ Number of patients } & \multirow{2}{*}{ Number of studies } & \multicolumn{2}{|c|}{ Heterogeneity test } \\
\hline & & & & $\mathrm{I}^{2}(\%)$ & $\mathrm{p}$ value \\
\hline \multicolumn{6}{|l|}{ Body weight } \\
\hline AR vs. LM & $1.66(0.63$ to 2.70$)$ & 496 & 10 & 55 & 0.02 \\
\hline AR vs. placebo & $1.15(0.67$ to 1.63$)$ & 833 & 14 & 65 & 0.0004 \\
\hline Combines therapies vs. PHA & $2.44(-1.98$ to 6.86$)$ & 80 & 1 & - & - \\
\hline Acupuncture vs. related therapies & $0.25(0.00$ to 0.49$)$ & 413 & 6 & 0 & 0.73 \\
\hline Combines therapies vs. acupuncture & $1.56(0.07$ to 3.05$)$ & 378 & 6 & 99 & $<0.00001$ \\
\hline \multicolumn{6}{|l|}{ BMI } \\
\hline AR vs. LM & $1.17(0.09$ to 2.26$)$ & 314 & 6 & 74 & 0.002 \\
\hline AR vs. placebo & $0.57(0.40$ to 0.74$)$ & 830 & 12 & 63 & 0.002 \\
\hline Combines therapies vs. PHA & $0.48(-0.90$ to 1.86$)$ & 80 & 1 & - & - \\
\hline Acupuncture vs. related therapies & $0.13(-0.06$ to 0.32$)$ & 325 & 5 & 0 & 0.8 \\
\hline Combines therapies vs. acupuncture & $0.77(-0.37$ to 1.92$)$ & 158 & 4 & 88 & $<0.00001$ \\
\hline
\end{tabular}

BMI: body mass index; LM: lifestyle modification; PHA: pharmacotherapy; AR: acupuncture and related therapies.

0.25to1.11), acupuncture (SMD: 1.28; 95\% CrI, 0.43to2.06), combination of acupuncture and related theories (SMD: 1.44; 95\% CrI, 0.64to2.38), and electroacupuncture (SMD: 0.60; 95\% CrI, 0.03tol.22). Also, the combination of acupuncture and related theories and acupuncture alone were both superior to the acupuncture combined lifestyle modification in their ability to reduce body mass index (SMD $=-1.76,95 \%$ $\mathrm{CrI}=-2.96$ to $-0.62 ; \mathrm{SMD}=-1.59,95 \% \mathrm{CrI}=-2.71$ to -0.34 ) (Table 5).

\subsection{Ranking}

3.5.1. Body Weight. Ranking of the different treatment methods was displayed Figure 3. The results suggested that, on the aspect of weight loss, combination of acupuncture and related therapies was ranked the optimal method, the best, $(88.7 \%)$, followed by moxibustion with warming needle $(87.8 \%)$, manual acupuncture (70.5\%), acupoint catgut embedding (ACE,62.1\%), auricular acupoint stimulation (AAS, $48.3 \%$ ), electro acupuncture (EA,46.3\%), pharmacotherapy (41.9\%), acupuncture combined lifestyle modification (AR+ LM,31.2\%), placebo acupuncture/sham acupuncture (16.8\%), and lifestyle modification (LM,6.4\%) which was ranked as the worst.

3.5.2. BMI. The results suggested that, on the aspect of BMI, combination of acupuncture and related therapies was ranked the optimal method, the best, $(90.2 \%)$, followed by manual acupuncture (83.3\%), pharmacotherapy (64.7\%), acupoint catgut embedding (58.6\%), auricular acupoint stimulation (55.7\%), electroacupuncture (52.1\%), placebo acupuncture/ sham acupuncture (25.1\%), acupuncture combined lifestyle modification (16.9\%), and lifestyle modification (3.4\%) which was ranked as the worst.

\subsection{Inconsistency Assessment}

3.6.1. Body Weight. The $\mathrm{Z}$ test illustrates the inconsistency of the NMA specifically (Appendix 4). For the inconsistency test outcome of BW, 95\% CI of 8 loops was included 0 , which reflected that no significant inconsistency was found. However, another 2 loops (ACE-Acupuncture-Combined therapies; ACE-Combined theories-EA) were found statistical inconsistency between direct and indirect comparisons.

3.6.2. BMI. For the inconsistency test outcome of body mass index, 95\% CI of all loops (acupuncture -combined theoriesEA; acupuncture-EA-placebo; AR+LM -EA- LM-placebo; ACE-acupuncture-EA; AAS-EA-LM; AAS-AR+LM-LMplacebo; AAS-EA-placebo) were included 0 , which reflected that no significant inconsistency was found.

3.7. Safety. Ten RCTs [26-28, 31, 32, 35, 36, 38, 39, 45] reported adverse events, while no major complications were noticed in all included studies. Three included studies [35, 36, 39] reported that no adverse effects were noted in both experimental group and placebo group. In one included RCT, there were two patients reporting mild ecchymosis and one abdominal discomfort case reported as adverse events after electroacupuncture treatment; no case was reported in the lifestyle modification group [27]. In another study, there were seven subjects in group auricular acupoint stimulation and two subjects in group placebo had mild tenderness [32].

\section{Discussion}

The aim of this study was to identify the efficacy and safety of acupoint stimulation therapy for obesity. In this NMA, the association of each acupuncture and related therapies with relative weight loss was compared by the combination of direct and indirect evidence from 34 RCTs in 2283 obese patients.

This study has three key findings. First, ranking graphs of the primary outcome suggested that the combination of acupuncture and related therapies was the most effective in losing weight and improving BMI. Second, compared with placebo or sham acupuncture, combination of acupuncture-related therapies, manual acupuncture, acupoint 


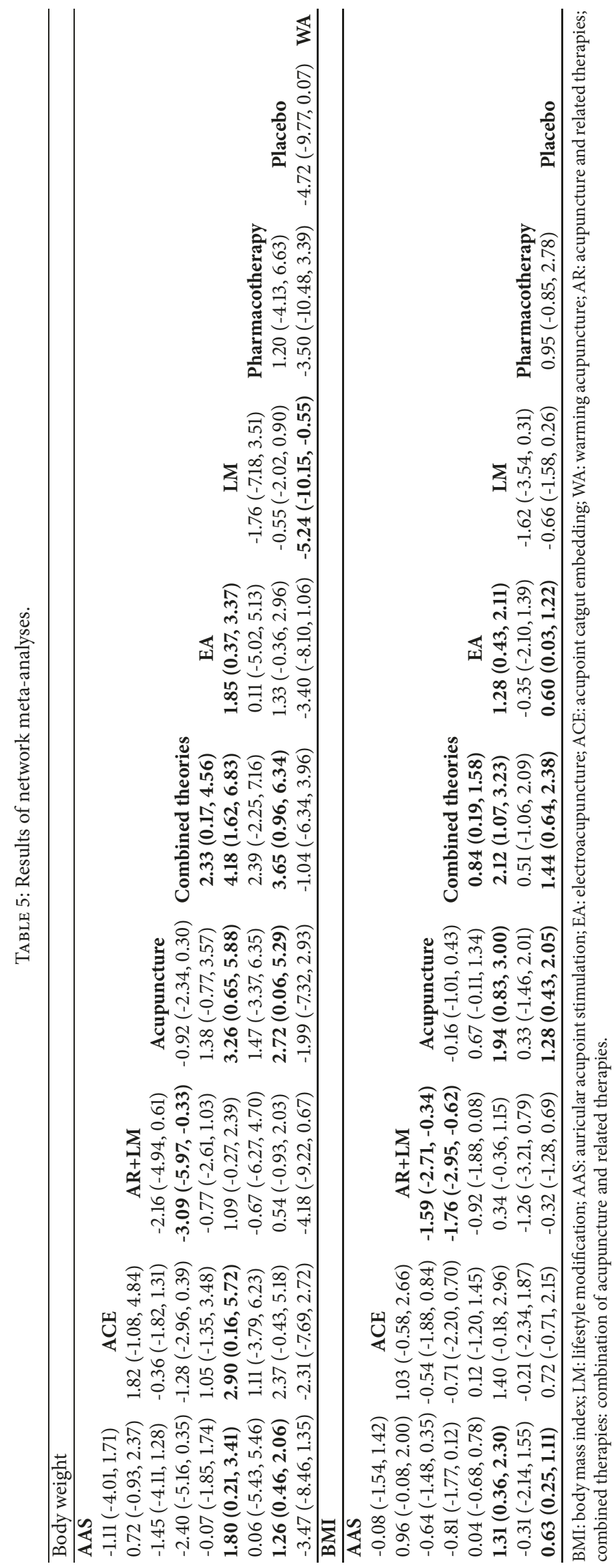



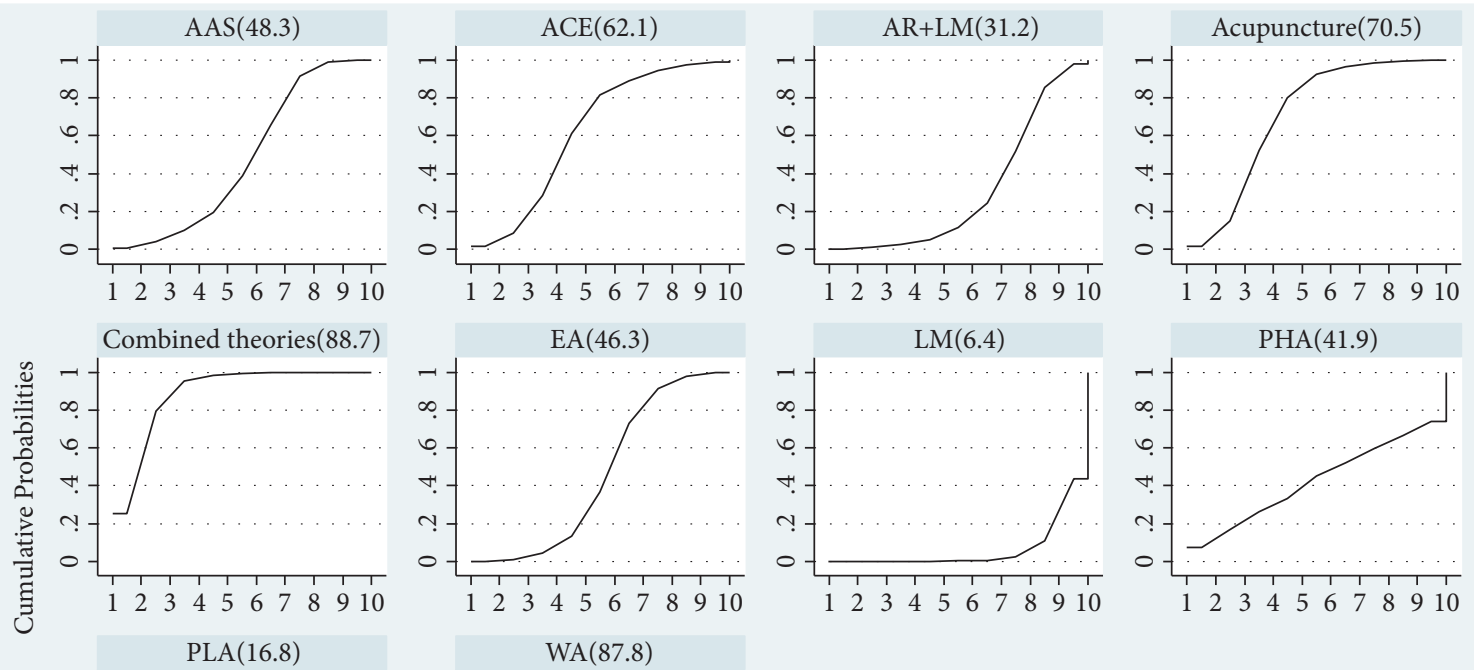

PHA(41.9)
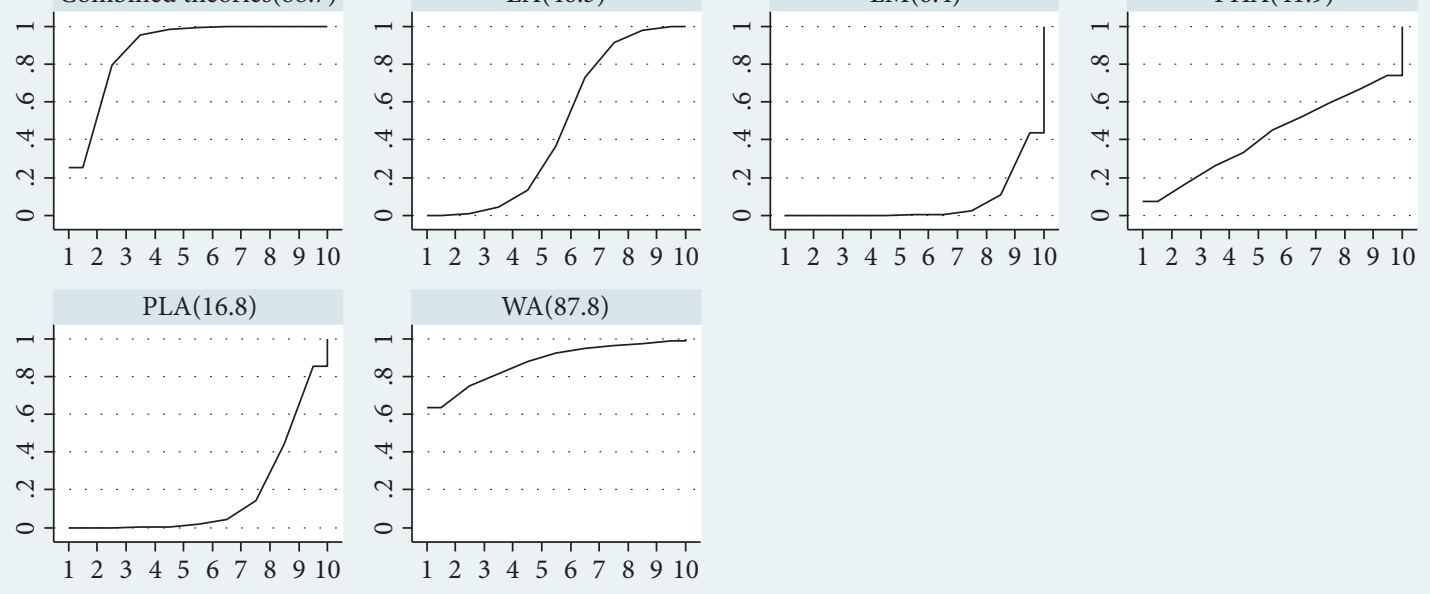

Graphs by Treatment

Rank

(a) Body weight
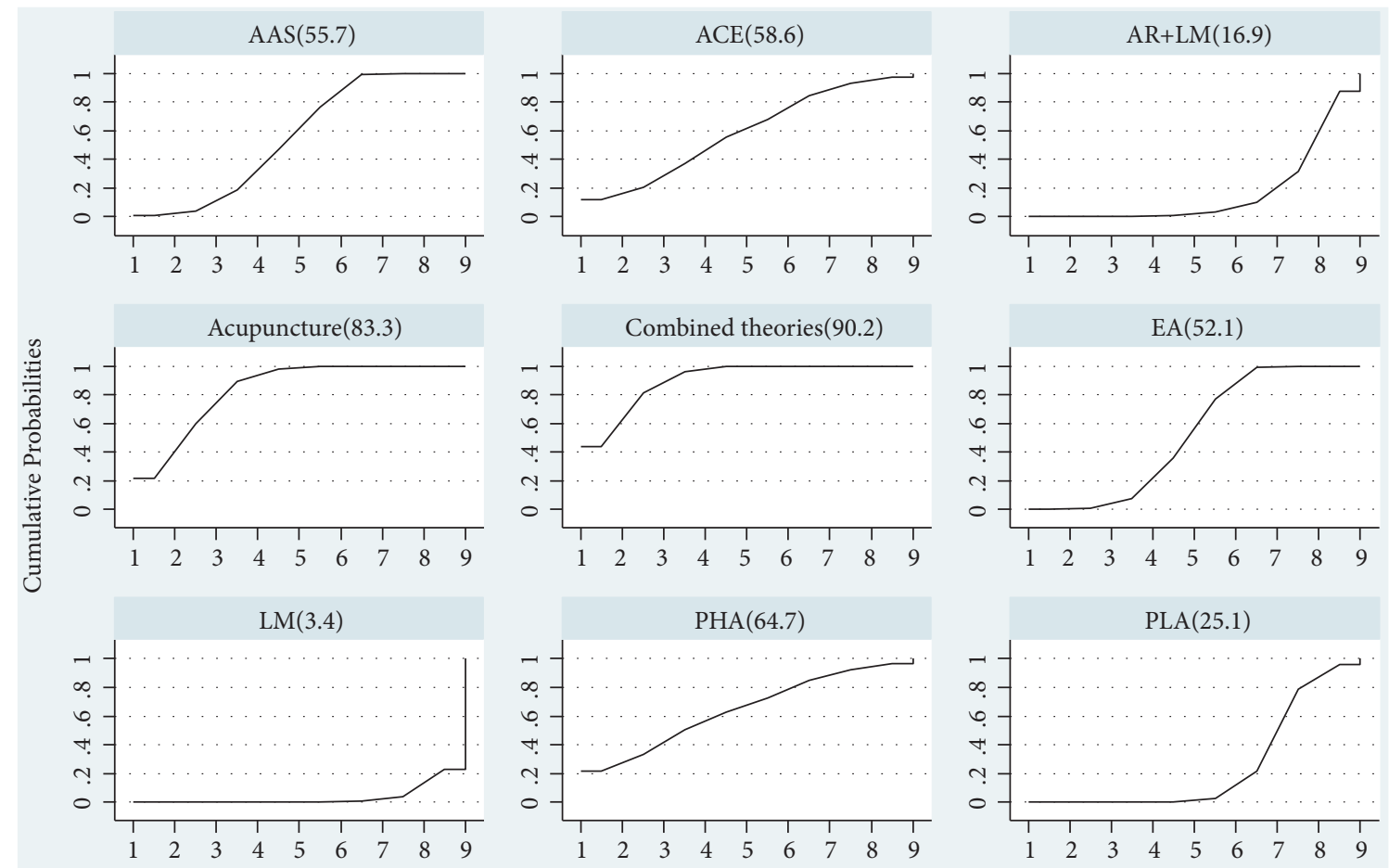

Graphs by Treatment
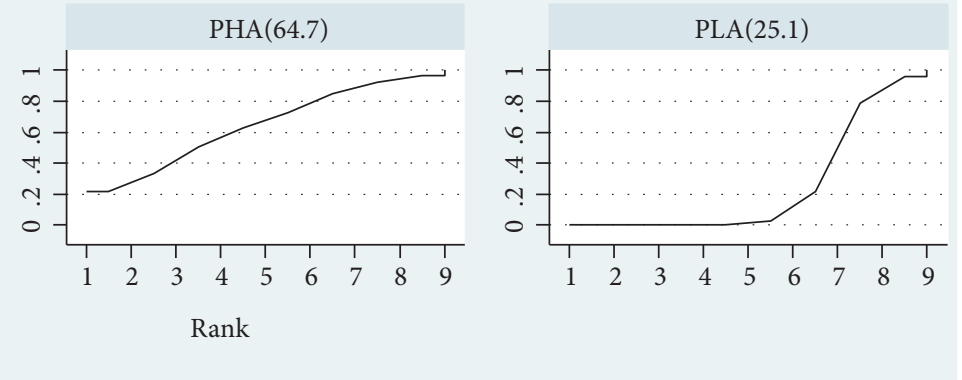

(b) Body mass index

FIGURE 3: Surface under the cumulative ranking curves. LM: lifestyle modification; AAS: auricular acupoint stimulation; EA: electroacupuncture; ACE: acupoint catgut embedding; WA: warming acupuncture; AR: acupuncture and related therapies; combined therapies: combination of acupuncture and related therapies; PLA: placebo; PHA: pharmacotherapy. 
catgut embedding, auricular acupuncture therapy, and electroacupuncture are all associated with higher odds of achieving weight loss. Third, combination of acupuncture and related therapies, manual acupuncture, pharmacotherapy, acupoint catgut embedding, auricular acupoint stimulation, and electroacupuncture were superior to lifestyle intervention.

Lifestyle modification, like diet intervention and physical activity, is recommended as safe and effective way to lose weight [60]. Results of direct and indirect evidence suggest acupuncture and related theories had significant beneficial effects in dealing with obesity compared with lifestyle modification. Both experimental and clinical data prove the efficacy of acupuncture for obesity [61]. Experimental data suggests that acupuncture exerts beneficial effects on weight loss $[62,63]$. The majority of clinical evidence suggests that acupuncture and related therapies reduced more weight than sham control group $[26,28,31,32]$, which are consistent with our results. Previous animal studies have observed that the expression of obesity-related peptides was upregulated in the hypothalamus after acupuncture treatment, which induced less food intake and weight loss $[62,64,65]$. Similarly, significant decreases in plasma leptin level were observed after EA treatment in obese patients [46]. With regard to insulin level, several experimental studies reported that EA can improve insulin sensitivity $[66,67]$. However, results from clinical trials regarding insulin levels are controversial. Cabioğlu MT reported that EA increased insulin level compared with control group [56], but Gucel F indicated that acupuncture decreased insulin level [46]. As to effects on lipid metabolism, acupuncture was reported to be effective in decreasing total cholesterol (TC), triglycerides (TG) and LDL-C concentrations $[68,69]$ of obese rat. Significant decreases in TC [55], TG [35], and LDL-C [55] were observed whereas no changes in HDL-C [55] levels were observed in clinical trials. Furthermore, experimental studies suggest that there was significant decrease in serum TNF $\alpha$ after EA [70]. Except for the noted mechanisms, EA can also induce white adipose tissue (WAT) browning via increasing uncoupling protein-1 (UCP1) gene expression [71].

This NMA has several attractive advantages. We focused on simple obesity patients without complication, which decreased the heterogeneity and improve the quality of this study. In addition, we compared acupuncture and acupuncture-related therapies with the first-line treatment for obesitylifestyle modification with a Bayesian framework. The rank test of effectiveness provides data to favour acupuncture and acupuncture-related therapies. Lastly, we conducted a comprehensive search and included all eligible studies. We compared five different acupuncture treatments (manual acupuncture; electro acupuncture; auricular acupoint stimulation; acupoint catgut embedding; moxibustion with warming needle) in the clinical effectiveness in treating patients with obesity.

However, this study has several limitations. First, we failed to evaluate the safety of each acupoint stimulation therapy due to the limited data in primary studies. Future trials should report adverse events clearly to improve the quality of study design. Second, unaddressed concerns still exist regarding the long-term effects of using acupuncture and acupuncture-related therapies on weight management in a clinical setting. The duration of acupuncture sessions and follow-up duration of most included trials ranging from four weeks to twelve weeks. Further clinical evaluation of acupuncture for obesity with longer follow-up appears warranted. Third, blinding of patients and researches was not applied among included studies and the included trials were mainly conducted in China, which may lead to publication bias [72]. Fourth, included study in our NMA lack of research compares the effectiveness between acupuncture, pharmacotherapy, and different types of combination of acupuncture. Further confirmatory comparative effectiveness trials should compare different types of combination of acupuncture. Except one study compared acupuncture and pharmacotherapy [29], additional research is needed to further explore. Finally, we use R-value to estimate the changes in standard deviations (SD), which might enlarge the SD compared with the originals values.

Overall, our results indicate that combination of acupuncture and related therapies ranks as the optimal method for reducing both weight and BMI. Further studies will clarify which combination of acupuncture and related therapies is better.

\section{Data Availability}

All data used to support the findings of this study are included within the supplementary information files.

\section{Conflicts of Interest}

All authors declare that they have no potential conflicts of interest.

\section{Authors' Contributions}

Yanji Zhang, Jia Li, and Guoyan Mo contributed equally to this work. Wei Huang, Zhongyu Zhou, and Yanji Zhang contributed to study design. Xianglin Chen, Hui Liu, and Teng Cai contributed to study selection. Xiangmin Tian, Teng Cai, Xian Zhang, and Xianglin Chen contributed to data collection and quality assessment. Figures $1-3$ were prepared by Jia Li and Jing Liu. Tables 1-5 were prepared by Guoyan Mo. Appendices 1-4 were prepared by Jia Li. Huisheng Yang and Teng Cai were responsible for technical and language support. All authors have read and approved the final manuscript.

\section{Acknowledgments}

This research was supported by the National Nature Science Foundation of China (nos. 81674081 and 81804165), 2015 Special Project in the TCM State Industry Administration of Traditional Chinese Medicine of the People's Republic of China (no. 201507003), Young Elite Scientist Sponsors Hip Program by CAST (no. 2017QNRC001), Hubei University of Traditional Chinese Medicine Acupuncture and Moxibustion 
Research Team Project (no. 2017ZXZ004), and Science and Technology Program of Hubei, China (no. 2016CFB221).

\section{Supplementary Materials}

Appendix 1: the PRISMA-NMA checklist treatment groups for included studies in a network meta-analysis. Appendix 2: search strategies for RCTs on acupuncture for obesity. Appendix 3: summaries of mean, standard difference, and sample size between treatment groups for included studies in a network meta-analysis. Appendix 4: inconsistency test. (Supplementary Materials)

\section{References}

[1] World Health Organization, "Fact Sheet Obesity and Overweight 2013," [WWW document], http://www.who.int/mediacentre/factsheets/fs311/en/\#, (accessed August 2014).

[2] W. T. Garvey, J. I. Mechanick, E. M. Brett et al., "American association of clinical endocrinologists and American college of endocrinology comprehensive clinical practice guidelines for medical care of patients with obesity," Endocrine Practice, vol. 22, pp. 1-203, 2016.

[3] C. L. Ogden, M. D. Carroll, B. K. Kit, and K. M. Flegal, "Prevalence of childhood and adult obesity in the United States, 2011-2012," The Journal of the American Medical Association, vol. 311, no. 8, pp. 806-814, 2014.

[4] W. Xu, H. Zhang, S. Paillard-Borg, H. Zhu, X. Qi, and D. Rizzuto, "Prevalence of overweight and obesity among Chinese adults: Role of adiposity indicators and age," Obesity Facts, vol. 9, no. 1, pp. 17-28, 2016.

[5] J. Cawley and C. Meyerhoefer, "The medical care costs of obesity: an instrumental variables approach," Journal of Health Economics, vol. 31, no. 1, pp. 219-230, 2012.

[6] R. Dalle Grave, S. Calugi, and M. El Ghoch, "Lifestyle modification in the management of obesity: Achievements and challenges," Eating and Weight Disorders, vol. 18, no. 4, pp. 339-349, 2013.

[7] R. Khera, M. H. Murad, A. K. Chandar et al., "Association of pharmacological treatments for obesity withweight loss and adverse events a systematic review and meta-analysis," Journal of the American Medical Association, vol. 315, no. 22, pp. 24242434, 2016.

[8] P. R. Schauer, S. R. Kashyap, K. Wolski et al., "Bariatric surgery versus intensive medical therapy in obese patients with diabetes," The New England Journal of Medicine, vol. 366, no. 17, pp. 1567-1576, 2012.

[9] S. Ikramuddin, J. Korner, W.-J. Lee et al., "Roux-en-Y gastric bypass vs intensive medical management for the control of type 2 diabetes, hypertension, and hyperlipidemia: the diabetes surgery study randomized clinical trial," The Journal of the American Medical Association, vol. 309, no. 21, pp. 2240-2249, 2013.

[10] L. O. Reis, W. J. Favaro, G. C. Barreiro et al., "Erectile dysfunction and hormonal imbalance in morbidly obese male is reversed after gastric bypass surgery: a prospective randomized controlled trial," International Journal of Andrology, vol. 33, no. 5, pp. 736-744, 2010.

[11] V. L. Gloy, M. Briel, D. L. Bhatt et al., "Bariatric surgery versus non-surgical treatment for obesity: a systematic review and meta-analysis of randomised controlled trials," British Medical Journal, vol. 347, no. 5, 2013.

[12] S. Fang, M. Wang, Y. Zheng, S. Zhou, and G. Ji, "Acupuncture and lifestyle modification treatment for obesity: a metaanalysis," American Journal of Chinese Medicine, vol. 45, no. 02, pp. 1-16, 2017.

[13] T.-L. Yeh, H.-H. Chen, T.-P. Pai et al., "The effect of auricular acupoint stimulation in overweight and obese adults: a systematic review and meta-analysis of randomized controlled trials," Evidence-Based Complementary and Alternative Medicine, vol. 2017, Article ID 3080547, 16 pages, 2017.

[14] T. Guo, Y. Ren, J. Kou, J. Shi, S. Tianxiao, and F. Liang, "Acupoint catgut embedding for obesity: systematic review and meta-analysis," Evidence-Based Complementary and Alternative Medicine, vol. 2015, Article ID 401914, 20 pages, 2015.

[15] A. Chaimani, J. P. T. Higgins, D. Mavridis, P. Spyridonos, and G. Salanti, "Graphical tools for network meta-analysis in STATA," PLoS ONE, vol. 8, no. 10, 2013.

[16] G. Salanti, C. Del Giovane, A. Chaimani, D. M. Caldwell, J. P. Higgins, and Y. Tu, "Evaluating the quality of evidence from a network meta-analysis," PLoS ONE, vol. 9, no. 7, 2014.

[17] B. Hutton, G. Salanti, D. M. Caldwell et al., “The PRISMA extension statement for reporting of systematic reviews incorporating network meta-analyses of health care interventions: Checklist and explanations," Annals of Internal Medicine, vol. 162, no. 11, pp. 777-784, 2015.

[18] The Cochrane Collaboration, "Cochrane handbook for systematic reviews of interventions," 2011, http://www.cochrane .org/training/cochrane-handbook.

[19] K. R. Abrams, C. L. Gillies, and P. C. Lambert, "Meta-analysis of heterogeneously reported trials assessing change from baseline," Statistics in Medicine, vol. 24, no. 24, pp. 3823-3844, 2005.

[20] J. P. T. Higgins, D. G. Altman, P. C. Gøtzsche et al., “The Cochrane Collaboration's tool for assessing risk of bias in randomised trials," British Medical Journal, vol. 343, no. 24, 2011.

[21] J. P. T. Higgins, S. G. Thompson, J. J. Deeks, and D. G. Altman, "Measuring inconsistency in meta-analyses," British Medical Journal, vol. 327, no. 7414, pp. 557-560, 2003.

[22] A. E. Ades, M. Sculpher, A. Sutton et al., "Bayesian methods for evidence synthesis in cost-effectiveness analysis," PharmacoEconomics, vol. 24, no. 1, pp. 1-19, 2006.

[23] G. Lu and A. E. Ades, "Combination of direct and indirect evidence in mixed treatment comparisons," Statistics in Medicine, vol. 23, no. 20, pp. 3105-3124, 2004.

[24] G. van Valkenhoef, G. Lu, B. de Brock, H. Hillege, A. E. Ades, and N. J. Welton, "Automating network meta-analysis," Research Synthesis Methods, vol. 3, no. 4, pp. 285-299, 2012.

[25] G. Salanti, A. E. Ades, and J. P. A. Ioannidis, "Graphical methods and numerical summaries for presenting results from multipletreatment meta-analysis: an overview and tutorial," Journal of Clinical Epidemiology, vol. 64, no. 2, pp. 163-171, 2011.

[26] D. B. Allison, K. Kreibich, and S. Heshka, "A randomised placebo-controlled clinical trial of an acupressure device for weight loss," International Journal of Obesity and Related Metabolic Disorders, vol. 19, no. 9, pp. 653-658, 1995.

[27] C.-H. Hsu, K.-C. Hwang, C.-L. Chao, J.-G. Lin, S.-T. Kao, and P. Chou, "Effects of electroacupuncture in reducing weight and waist circumference in obese women: a randomized crossover trial," International Journal of Obesity, vol. 29, no. 11, pp. 13791384, 2005. 
[28] D. Richards and J. Marley, "Stimulation of auricular acupuncture points in weight loss," Australian Family Physician, vol. 2, no. 27, pp. S73-S77, 1998.

[29] L. He, X.-L. Gao, H.-X. Deng, and Y.-X. Zhao, "Effects of acupuncture on body mass index and waist-hip ratio in the patient of simple obesity," Chinese Acupuncture \& Moxibustion, vol. 28, no. 2, pp. 95-97, 2008 (Chinese).

[30] L. Li and Z.-Y. Wang, "Clinical therapeutic effects of body acupuncture and ear acupuncture on juvenile simple obesity and effects on metabolism of blood lipids," Chinese Acupuncture \& Moxibustion, vol. 26, no. 3, pp. 173-176, 2006.

[31] J. Tong, J. X. Chen, Z. Q. Zhang et al., "Clinical observation on simple obesity treated by acupuncture," Chinese Acupuncture \& Moxibustion, vol. 31, no. 8, pp. 679-701, 2011.

[32] C.-H. Hsu, C.-J. Wang, K.-C. Hwang, T.-Y. Lee, P. Chou, and H.H. Chang, "The effect of auricular acupuncture in obese women: a randomized controlled trial," Journal of Women's Health, vol. 18, no. 6, pp. 813-818, 2009.

[33] C. H. Hsieh, "The effects of auricular acupressure on weight loss and serum lipid levels in overweight adolescents," American Journal of Chinese Medicine, vol. 38, no. 4, pp. 675-682, 2010.

[34] C. H. Hsieh, T.-J. Su, Y.-W. Fang, and P.-H. Chou, "Effects of auricular acupressure on weight reduction and abdominal obesity in asian young adults: a randomized controlled trial," American Journal of Chinese Medicine, vol. 39, no. 3, pp. 433440, 2011.

[35] H. Abdi, P. Abbasi-Parizad, B. Zhao et al., "Effects of auricular acupuncture on anthropometric, lipid profile, inflammatory, and immunologic markers: a randomized controlled trial study," The Journal of Alternative and Complementary Medicine, vol. 18, no. 7, pp. 668-677, 2012.

[36] M. Darbandi, S. Darbandi, M. G. Mobarhan et al., "Effects of auricular acupressure combined with low-Calorie diet on the leptin hormone in obese and overweight iranian individuals," Acupuncture in Medicine, vol. 30, no. 3, pp. 208-213, 2012.

[37] W. He, Z. Zhou, J. Li, L. Wang, B. Zhu, and G. Litscher, "Auricular acupressure plus exercise for treating primary obese women: a randomized controlled clinical trial," Medical Acupuncture, vol. 24, no. 4, pp. 227-232, 2012.

[38] C. Y. Lien, L. L. Liao, P. Chou, and C. H. Hsu, "Effects of auricular stimulation on obese women: a randomized, controlled clinical trial," European Journal of Integrative Medicine, vol. 4, no. 1, pp. e45-e53, 2012.

[39] M. Darbandi, S. Darbandi, A. A. Owji et al., "Auricular or body acupuncture: Which one is more effective in reducing abdominal fat mass in Iranian men with obesity: A randomized clinical trial," Journal of Diabetes and Metabolic Disorders, vol. 13, no. 1, 2014.

[40] M.-L. Yeh, N.-F. Chu, M.-Y. F. Hsu, C.-C. Hsu, and Y.-C. Chung, "Acupoint stimulation on weight reduction for obesity: a randomized sham-controlled study," Western Journal of Nursing Research, vol. 37, no. 12, pp. 1517-1530, 2015.

[41] F. Chen, S. Wu, and Y. Zhang, "Effect of acupoint catgut embedding on TNF-alpha and insulin resistance in simple obesity patients," Acupuncture Research, vol. 32, no. 1, pp. 49-52, 2007.

[42] L.-C. Huang and W.-Y. Pan, "Comparation of effect and costbenefit analysis between acupoint catgut-embedding and electroacupuncture on simple obesity," Chinese Acupuncture \& Moxibustion, vol. 31, no. 10, pp. 883-886, 2011.

[43] C.-L. Tang, D.-C. Dai, G.-F. Zhao, W.-F. Zhu, and L.-F. Mei, "Clinical observation on electroacupuncture combined with catgut implantation at acupoints for treatment of simple obesity of heart and spleen deficiency type," Chinese Acupuncture \& Moxibustion, vol. 29, no. 9, pp. 703-707, 2009.

[44] Y. Shi, L.-S. Zhang, C. Zhao, and C.-Q. He, "Comparison of therapeutic effects of acupuncture-cupping plus acupoint catgut embedding and electroacupuncture on simple obesity of stomach and intestine excess-heat type," Chinese Acupuncture \& Moxibustion, vol. 26, no. 8, pp. 547-550, 2006.

[45] C.-H. Hsu, K.-C. Hwang, C.-L. Chao, H.-H. Chang, and P. Chou, "Electroacupuncture in obese women: A randomized, controlled pilot study," Journal of Women's Health, vol. 14, no. 5, pp. 434-440, 2005.

[46] F. Gucel, B. Bahar, C. Demirtas, S. Mit, and C. Cevik, "Influence of acupuncture on leptin, ghrelin, insulin and cholecystokinin in obese women: a randomised, sham-controlled preliminary trial," Acupuncture in Medicine, vol. 30, no. 3, pp. 203-207, 2012.

[47] L. Deng, Z. Lun, X. Ma, and J. Zhou, "Clinical observation on regulating the three energizer by acupoint catgut embedding combined with abdominal acupuncture in treating abdominal obesity: a randomized controlled trial," World Journal of Acupuncture - Moxibustion, vol. 24, no. 4, pp. 29-34, 2014.

[48] N. E. Hassan, S. A. El-Masry, S. M. Elshebini et al., "Comparison of three protocols: Dietary therapy and physical activity, acupuncture, or laser acupuncture in management of obese females," Macedonian Journal of Medical Sciences, vol. 7, no. 2, pp. 191-197, 2014.

[49] J. He, X. Zhang, Y. Qu et al., "Effect of combined manual acupuncture and massage on body weight and body mass index reduction in obese and overweight women: a randomized, shortterm clinical trial," Journal of Acupuncture and Meridian Studies, vol. 8, no. 2, pp. 61-65, 2015.

[50] Y. L. Wang, X. Cao, Z. C. Liu, and B. Xu, "Observation on the therapeutic effect of electroacupuncture on simple obesity of gastrointestinal heat pattern/syndrome," World Journal of Acupuncture - Moxibustion, vol. 23, no. 2, pp. 1-5, 2013.

[51] S. Yeo, K. S. Kim, and S. Lim, "Randomised clinical trial of five ear acupuncture points for the treatment of overweight people," Acupuncture in Medicine, vol. 32, no. 2, pp. 132-138, 2014.

[52] T. W. Bu, X. L. Tian, S. J. Wang et al., "Comparison and analysis of therapeutic effects of different therapies on simple obesity," Chinese Acupuncture \& Moxibustion, vol. 27, no. 5, pp. 337-340, 2007.

[53] Y. Shi, L.-S. Zhang, C. Zhao, and X.-Y. Zuo, "Controlled study of needle warming therapy and electroacupuncture on simple obesity of spleen deficiency type," Chinese Acupuncture \& Moxibustion, vol. 25, no. 7, pp. 465-467, 2005.

[54] J.-J. Yang, H.-J. Xing, H.-L. Xiao, Q. Li, M. Li, and S.-J. Wang, "Effects of acupuncture combined with diet adjustment and aerobic exercise on weight and waist-hip ratio in simple obesity patients," Chinese Acupuncture \& Moxibustion, vol. 30, no. 7, pp. 555-558, 2010.

[55] M. T. Cabığlu and N. Ergene, "Electroacupuncture therapy for weight loss reduces serum total cholesterol, triglycerides, and LDL cholesterol levels in obese women," American Journal of Chinese Medicine, vol. 33, no. 4, pp. 525-533, 2005.

[56] M. T. Cabioglu and N. Ergene, "Changes in levels of serum insulin, C-peptide and glucose after electroacupuncture and diet therapy in obese women," American Journal of Chinese Medicine, vol. 34, no. 3, pp. 367-376, 2006.

[57] M. T. Cabioglu, N. Gündoğan, and N. Ergene, "The efficacy of electroacupuncture therapy for weight loss changes plasma 
lipoprotein A, Apolipoprotein A and Apolipoprotein B levels in obese women," American Journal of Chinese Medicine, vol. 36, no. 6, pp. 1029-1039, 2008.

[58] S. Darbandi, M. Darbandi, and P. Mokarram, "Effects of body electroacupuncture on plasma leptin concentrations in obese and overweight people in Iran: a randomized controlled trial," Alternative Therapies in Health and Medicine, vol. 19, no. 2, pp. 24-31, 2013.

[59] S. Fogarty, L. Stojanovska, D. Harris, C. Zaslawski, M. L. Mathai, and A. J. McAinch, "A randomised cross-over pilot study investigating the use of acupuncture to promote weight loss and mental health in overweight and obese individuals participating in a weight loss program," Eating and Weight Disorders, vol. 20, no. 3, pp. 379-387, 2015.

[60] W. T. Garvey, J. I. Mechanick, E. M. Brett et al., "American association of clinical endocrinologists and American college of endocrinology comprehensive clinical practice guidelines for medical care of patients with obesity: Executive summary," Endocrine Practice, vol. 22, no. 7, pp. 842-884, 2016.

[61] M. Belivani, C. Dimitroula, N. Katsiki, M. Apostolopoulou, M. Cummings, and A. I. Hatzitolios, "Acupuncture in the treatment of obesity: a narrative review of the literature," Acupuncture in Medicine, vol. 31, no. 1, pp. 88-97, 2013.

[62] D.-R. Tian, X.-D. Li, F. Wang et al., "Up-regulation of the expression of cocaine and amphetamine-regulated transcript peptide by electroacupuncture in the arcuate nucleus of dietinduced obese rats," Neuroscience Letters, vol. 383, no. 1-2, pp. 17-21, 2005.

[63] N. Tian, F. Wang, D.-R. Tian et al., "Electroacupuncture suppresses expression of gastric ghrelin and hypothalamic NPY in chronic food restricted rats," Peptides, vol. 27, no. 9, pp. 23132320, 2006.

[64] F. Wang, D. R. Tian, P. Tso, and J. S. Han, "Arcuate nucleus of hypothalamus is involved in mediating the satiety effect of electroacupuncture in obese rats," Peptides, vol.32, no. 12, pp. 23942399, 2011.

[65] Z. Liu, F. Sun, J. Su et al., "Study on action of acupuncture on ventromedial nucleus of hypothalamus in obese rats," Journal of Traditional Chinese Medicine, vol. 21, no. 3, pp. 220-224, 2001.

[66] J.-G. Lin, W.-C. Chen, C.-L. Hsieh et al., "Multiple sources of endogenous opioid peptide involved in the hypoglycemic response to $15 \mathrm{~Hz}$ electroacupuncture at the Zhongwan acupoint in rats," Neuroscience Letters, vol. 366, no. 1, pp. 39-42, 2004.

[67] Y. Higashimura, R. Shimoju, H. Maruyama, and M. Kurosawa, "Electro-acupuncture improves responsiveness to insulin via excitation of somatic afferent fibers in diabetic rats," Autonomic Neuroscience: Basic and Clinical, vol. 150, no. 1-2, pp. 100-103, 2009.

[68] S. Wang, Q. Li, Y. She et al., "Effect of electroacupuncture on metabolism of lipids in rats of obesity induced by sodium glutamate," Chinese Acupuncture \& Moxibustion, vol. 25, no. 4, pp. 269-271, 2005.

[69] S. J. Wang, H. Z. Xu, and H. L. Xiao, "Effect of high-frequency electroacupuncture on lipid metabolism in obesity rats," Acupuncture Research, vol. 33, no. 3, pp. 154-158, 2008.

[70] H. Yang, Y. Li, L. Cheng, and J.-S. He, "Effect of electroacupuncture and diet adjusting on insulin resistance in rats with nutrition obesity," Journal of Chinese Integrative Medicine, vol. 5, no. 5, pp. 546-549, 2007.

[71] W. Shen, Y. Wang, S.-F. Lu et al., "Acupuncture promotes white adipose tissue browning by inducing UCP1 expression on DIO mice," BMC Complementary and Alternative Medicine, vol. 14, no. 1, pp. 54-59, 2014.

[72] M. J. Page, J. P. Higgins, G. Clayton et al., "Empirical evidence of study design biases in randomized trials: systematic review of meta-epidemiological studies," PLoS ONE, vol. 11, no. 7, pp. 5-9, 2016. 


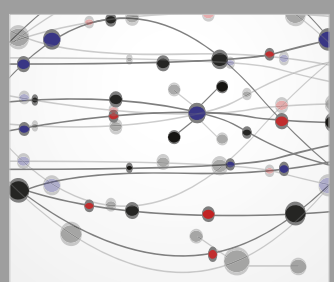

The Scientific World Journal
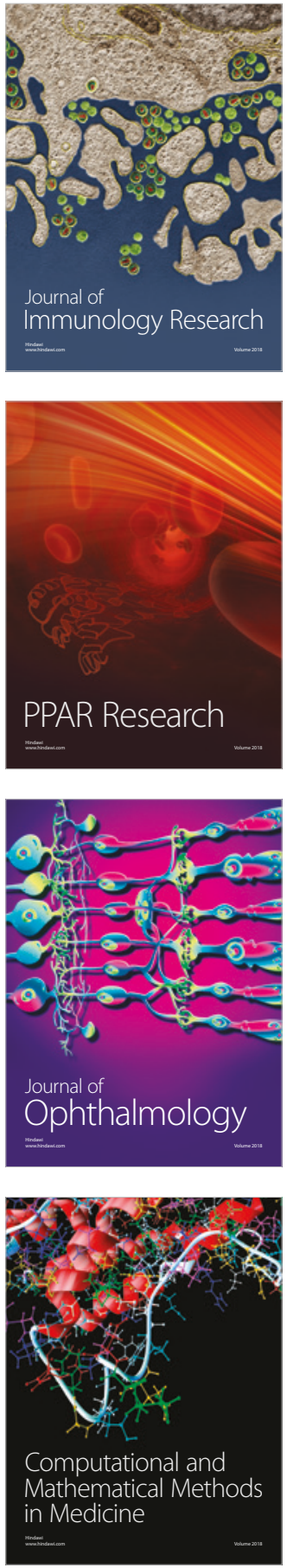

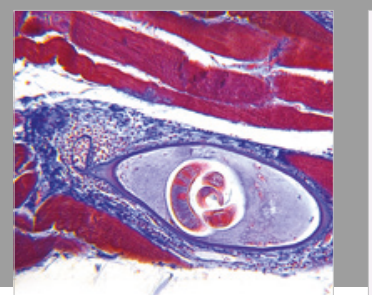

Gastroenterology Research and Practice

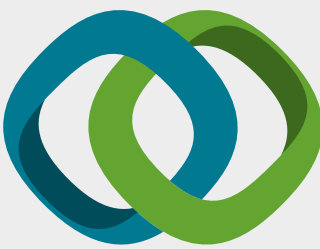

\section{Hindawi}

Submit your manuscripts at

www.hindawi.com
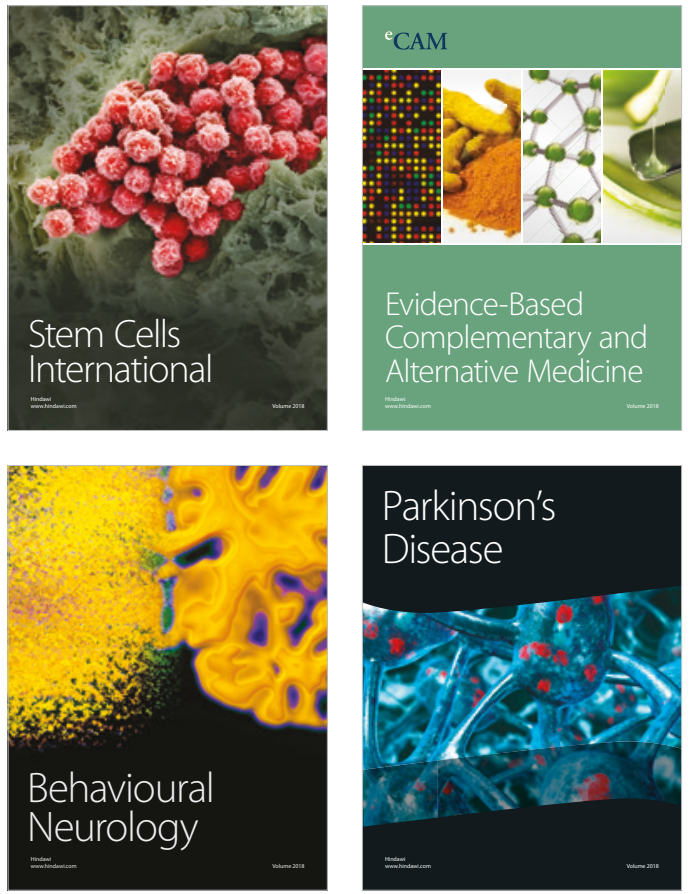

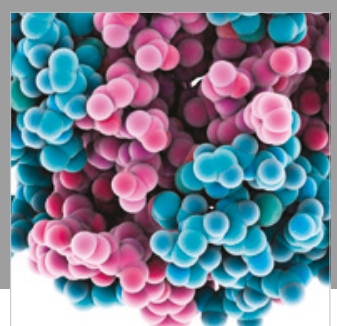

ournal of

Diabetes Research

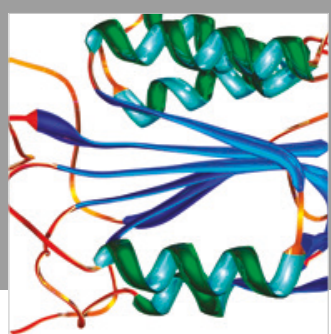

Disease Markers
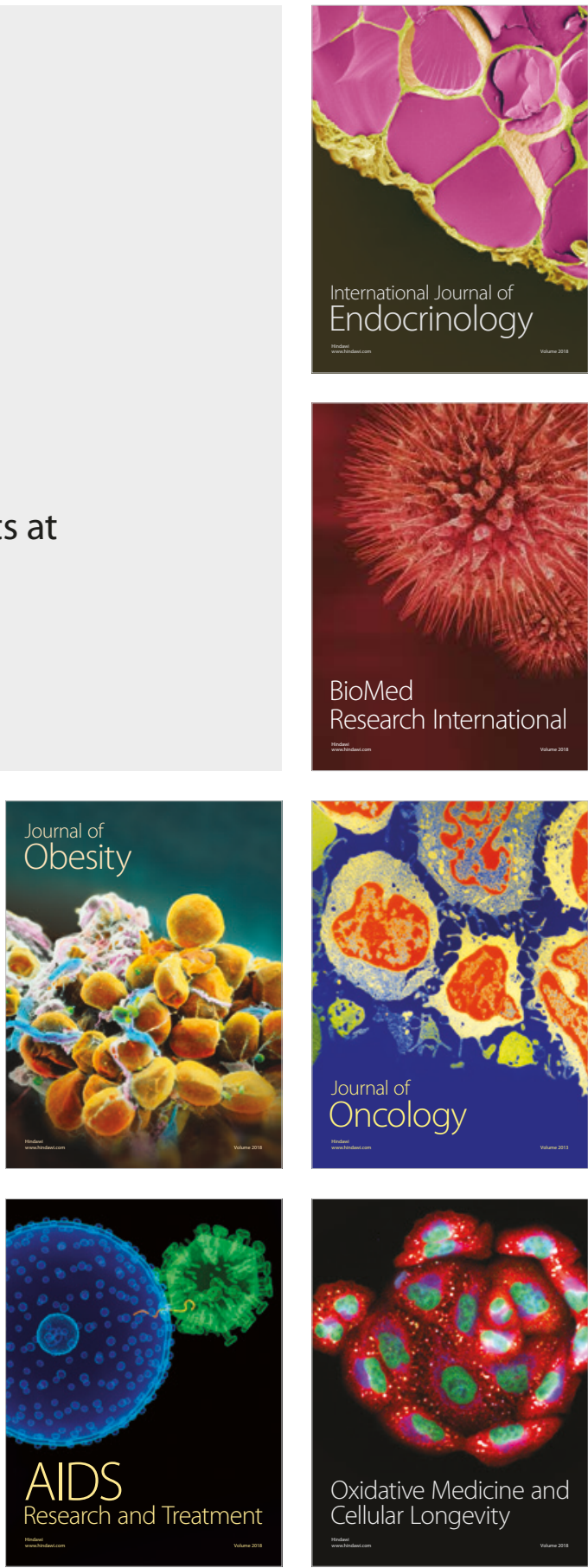